\title{
Brain acetylcholinesterase activity is markedly reduced in dominantly-inherited olivopontocerebellar atrophy
}

\author{
STEPHEN J KISH, LAWRENCE SCHUT, JERRY SIMMONS, JOSEPH GILBERT, \\ LI-JAN CHANG, MICHELLE REBBETOY
}

From the Human Brain Laboratory, Clarke Institute of Psychiatry, Toronto, Canada; Veterans Administration Hospital, Minneapolis, USA; Victoria Hospital, London, Canada; and University of South Dakota, Sioux Falls, USA

SUMMARY The activity was measured of the acetylcholine catabolising enzyme acetylcholinesterase (AChE) in brain after necropsy of seven patients from one established pedigree with dominantly-inherited olivopontocerebellar atrophy (OPCA), a cerebellar ataxia disorder in which neuropathological changes are assumed to be primarily restricted to cerebellum, lower brain stem and spinal cord. Mean AChE activity was significantly reduced in cerebral $(-51 \%$ to $65 \%)$ and cerebellar $(-47 \%)$ cortex with a less severe change $(-37 \%)$ in the hippocampus. The magnitude of the enzyme reduction in cerebral cortex was equal to or greater than that reported in brain of demented Alzheimer's and Parkinson's disease patients having loss of AChE-containing nucleus basalis cholinergic neurons. It is concluded that the data provide additional biochemical evidence suggesting a cerebral cortical cholinergic denervation in OPCA.

Much circumstantial evidence suggests that an abnormality of the brain cholinergic system may underlie the cognitive dysfunction of Alzheimer's disease. In this regard, reduced activity of the cholinergic enzymes choline acetyltransferase (ChAT, E.C. 2.3.1.6. ${ }^{1-3}$ ) and acetylcholinesterase (AChE, E.C. 3.1.1.7. ${ }^{4-6}$ ) as well as decreased acetylcholine synthesis ${ }^{7}$ have been convincingly demonstrated in necropsied and/or biopsied Alzheimer's disease brain. The results of neuropathological studies in Alzheimer's disease have provided a morphological explanation for the cholinergic enzyme reduction, namely, a loss of cholinergic neurons emanating from the nucleus basalis-septum-diagonal band of Broca brain region, and terminating in cerebral cortex and limbic subcortical areas. ${ }^{8}$ Taken together, the above data provide circumstantial evidence for the cholinergic hypothesis of Alzheimer's dementia.

Should the cognitive impairment of Alzheimer's

Address for reprint requests: Stephen J Kish, Human Brain Lab, Clarke Institute of Psychiatry, 250 College Street, Toronto, Ontario M5T 1R8, Canada.

Received 4 August 1987 and in revised form 7 October 1987 Accepted 12 October 1987 disease dementia be fundamentally due to a brain cholinergic dysfunction ${ }^{9}$ a cholinergic reduction in brain of individuals afflicted with non-Alzheimer's disease neurodengenerative disorders would also be expected to be associated with dementia of Alzheimer's type. In this regard, Perry and coworkers ${ }^{5}$ have recently reported a good correlation between the degree of cognitive dysfunction and extent of cortical ChAT loss in demented Parkinson's disease patients. Interestingly, however, in this study the severity of the cognitive impairment in the demented Parkinson's disease patients was, on average, less than that observed in Alzheimer's disease dementia despite having cortical cholinergic (ChAT and $\mathrm{AChE}$ ) reduction of similar magnitude. Moreover, the majority of the Parkinson's disease patients examined did not have any of the cognitive deficits (aphasia, apraxia and agnosia) often associated with Alzheimer's disease.

We have recently described a cerebral cortical reduction in the activity of the cholinergic marker synthetic enzyme ChAT in the brain of patients from five families afflicted with another non-Alzheimer's disease neurodegenerative disorder, namely, 
dominantly-inherited olivopontocerebellar atrophy $\left(\mathrm{OPCA},{ }^{10}\right]$, a cerebellar ataxia disorder in which neuropathological changes are assumed to be restricted to cerebellum, lower brain stem, and spinal cord. ${ }^{11}$ Although dementia is not considered to be a clinical feature in patients from this OPCA pedigree ${ }^{12}$ we discovered that the magnitude of the cortical ChAT reduction in OPCA was comparable with that reported in Alzheimer's disease brain.

The present investigation was undertaken to clarify further biochemically the status of the cholinergic system in OPCA brain through examination, in the largest of the five families previously studied (Schut), of the behaviour of a second cholinergic enzyme, AChE. Although AChE is localised in both cholinergic and non-cholinergic neurons, ${ }^{13}$ a significant loss of cholinergic neurons should be associated with decreased AChE enzyme activity. Thus, loss of basal forebrain (nucleus basalis-septum) cholinergic innervation to cerebral cortex or limbic brain resulting from either experimental lesions ${ }^{14} 15$ or human neurodegenerative condition (Alzheimer's disease ${ }^{14-61617}$, elderly Down's Syndrome ${ }^{18}$,

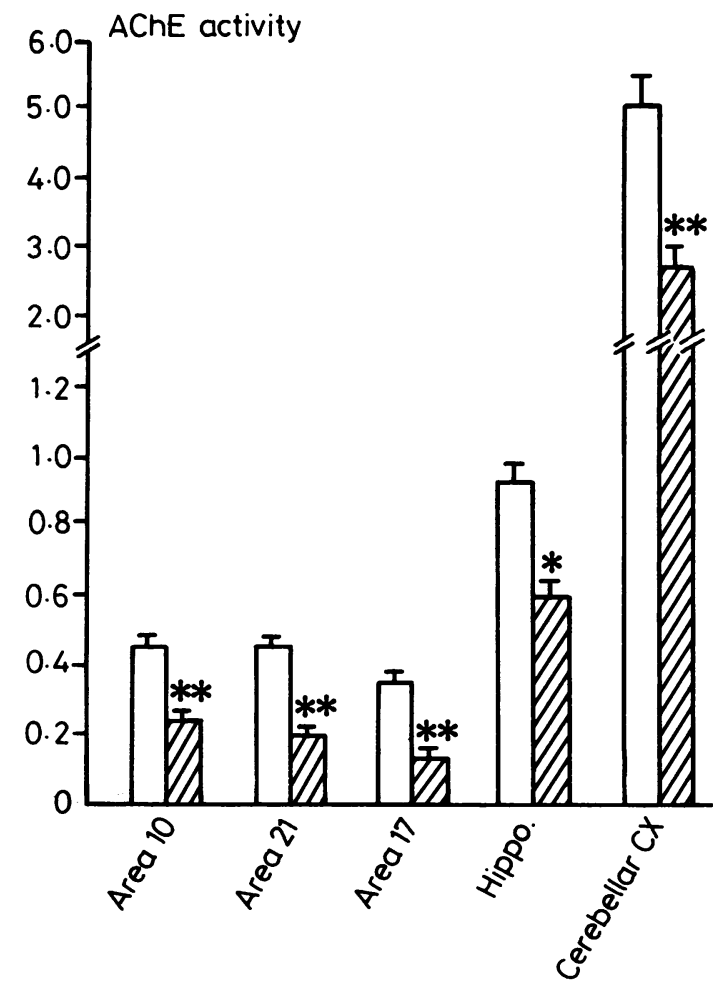

Fig Acetylcholinesterase (AChE, mmol/g protein/hour) and cholineacetyltransferase (ChAT, nmol/mg protein/10 min) activity in brain of controls (solid bar) and OPCA (cross hatched bar) patients. Cerebral cortical areas are frontal (area 10), temporal (area 21) and occipital (area 17). Values represent mean, S.E. ${ }^{*} p<0.05 ;{ }^{* *} p<0.001$; Student's two-tailed $t$ test.

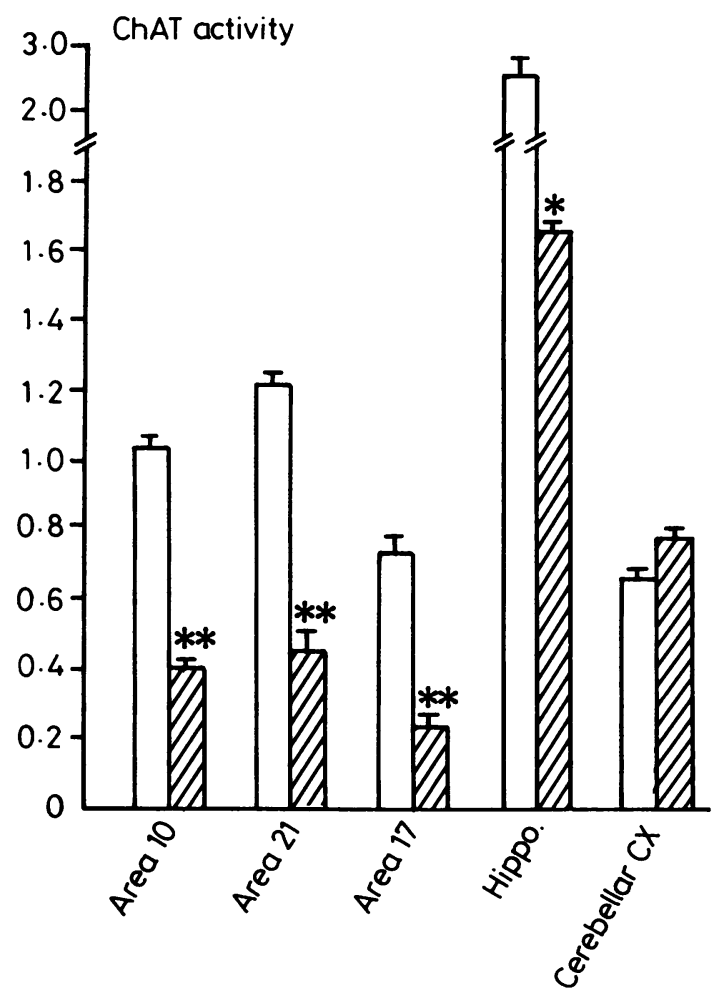

Parkinson's disease ${ }^{519}$ ) is consistently associated with reduced $\mathrm{AChE}$ activity in the terminal regions. Studies of brain AChE in human neurodegenerative conditions may also be of interest in view of the recent evidence that AChE, in addition to its role in acetylcholine catabolism, may have non-cholinergic physiological actions including peptide metabolism and regulation of nigrostriatal neuron activity. ${ }^{20}$

\section{Patients and methods}

Brain was obtained at necropsy from seven patients from the Schut kindred. This kindred, which now spans eight generations, was originally described by Gray and Oliver ${ }^{21}$ and has been continuously followed. The autosomal dominant locus has been localised to a region 15 centimorgans telomeric of HLA-A on the short arm of chromosome $6 .^{2223}$ The diagnosis of dominantly-inherited OPCA was made on the basis of clinical signs (limb and gait ataxia, dysarthria and dysphagia), positive family history, and characteristic histological changes in cerebellum, pons and inferior olive. ${ }^{24}$ Histological analyses of extracerebellar brain areas conducted on two (only) of the seven OPCA patients revealed an absence of senile plaques, neurofibrillary tangles, or 
significant cell loss in cerebral cortex. Some brain ChAT data on three of the seven patients have been recently described. ${ }^{10}$ Necropsy brains from nine control subjects without neurological or psychiatric illness were also obtained. Mean ages for the two groups (controls: 39, 4 years; OPCA: 35,2 years (mean, SE)) did not differ significantly ( $\mathrm{p}>0.05$, Students' two-tailed $t$ test). The mean interval between death and freezing of the brain at $-80^{\circ} \mathrm{C}$ did not differ significantly for the two groups (controls: 12, 2 hours; OPCA: 9, 2; p > 0.05).

Total AChE was determined by minor modifications of the procedures of Ellman et $a l^{25}$ and Bonham et $a l^{26}$ employing $1 \mathrm{mM}$ acetyl-beta-methylthio-choline as substrate in the presence of $0.01 \mathrm{mM}$ iso-OMPA (Sigma) a specific inhibitor of butyrylcholinesterase. Blanks contained 0.01 mM BW284C51 (Sigma), a specific inhibitor of AChE. ChAT activity was determined by the radiochemical method of Fonnum. ${ }^{27}$

\section{Results}

As shown in the fig the activity of AChE in control brain varied among the different areas examined, with the cerebellar cortex having about ten-fold higher activity as compared with cerebral cortex.

When compared with the controls mean AChE activity in OPCA brain was markedly and statistically significantly reduced in cerebral $(-51 \%$ to $-65 \%)$ and cerebellar $(-47 \%)$ cortex with the hippocampus less severely affected $(-37 \%)$.

Mean ChAT activity in OPCA was markedly and statistically significantly reduced by 65 to $72 \%$ in cerebral cortex with the hippocampus again being less affected $(-36 \%)$. In cerebellar cortex ChAT levels were nonsignificantly $(p>0.05)$ elevated by $17 \%$.

\section{Discussion}

\section{Control levels and distribution}

In control human brain regions the mean activity of (total) AChE was similar in magnitude to the levels reported by some groups 51628 but was about onetenth the values obtained by Davies. ${ }^{1}$ In agreement with the recent findings of Atack and co-workers ${ }^{28}$ enzyme activity in the cere bellar cortex was about ten-fold higher than cerebral cortex whereas hippocampal values were about twice that of cerebral cortex.

\section{Cerebellum}

Although the physiological function of acetylcholine in cerebellum is not well understood, an immunohistochemical study in human brain indicates that ChAT, the acetylcholine synthesizing enzyme, appears to be exclusively localised in mossy fibres (possibly originating in the pons) which terminate in the granule cell layer. ${ }^{2930}$ In contradistinction, the results of histochemical staining studies of the acetyl- choline catabolising enzyme $\mathrm{AChE}$ demonstrate a more diffuse distribution in both molecular and granule cell layers of the cerebellar cortex with possible localisation to mossy fibres, Golgi cells, and (especially in the human) granule cell terminals. ${ }^{293132}$ Our observation in OPCA of a $45 \%$ reduction in AChE in cerebellar cortical homogenates is in agreement with neuropathological findings in a single case with hereditary OPCA demonstrating markedly decreased AChE activity in the molecular layer of cerebellar cortex. ${ }^{32}$ In view of the possible localisation of $\mathrm{AChE}$ to granule cell terminals in the molecular layer, our data are also consistent with the histological observations of Landis and co-workers ${ }^{33}$ demonstrating degenerative changes in granule cells in (biopsied) cerebellar cortex of patients from our pedigree.

In contrast to AChE, the activity of ChAT was not significantly different from the controls in OPCA cerebellar cortex. In a previous study of OPCA, cerebellar ChAT levels were reported to be variable with values ranging from 24 to $168 \%$ of controls. ${ }^{34}$ Since in OPCA there is marked shrinkage of the cerebellum, ${ }^{111224}$ preservation of afferent terminals to cerebellar cortex should result in artifactually abovenormal levels of neurotransmitter indices localised to cortical nerve endings when expressed on a concentration basis. Thus, our observation of normal or only slightly elevated ChAT activity in the atrophic cerebellar cortex suggests an actual loss of ChATcontaining (possibly mossy fibre) terminals.

\section{Extracerebellar brain areas}

To our knowledge this study describes, for the first time, the behaviour of AChE in extracerebellar areas of OPCA brain. Our observation of markedly reduced cortical ChAT activity in a total of seven members of the Schut pedigree confirms and extends our original report (ref. 10, $\mathrm{n}=3$ ).

The biochemical demonstration in cerebral cortex and, to a lesser extent, in hippocampus, of reduced activity of both AChE and ChAT strengthens the notion that an actual loss of cholinergic neurones containing these acetylcholine metabolising enzymes is present in OPCA brain. In this regard, in a recent neuropathological study of the basal forebrain cholinergic system in brain of a single patient from a different OPCA family (probable dominant inheritance) a significant $(>60 \%)$ neuronal loss was observed in the magnocellular population of the basal nucleus of Meynert, ${ }^{35}$ the brain region which provides the major source of cholinergic innervation ascending to neocortex. ${ }^{36}$ With respect to the present study, in a preliminary (qualitative) histological investigation of the nucleus basalis of patients from the Schut OPCA pedigree we have also observed a marked 
reduction in number of $\mathrm{AChE}$ staining magnocellular neurons with a less severe involvement of the smaller neurons (Kish and Robitaille, unpublished observations; quantitative morphometric studies in progress).

The magnitude of the cortical AChE reduction in OPCA ( -51 to $-65 \%)$ was equal to or greater than the enzyme deficit reported in brain of demented patients with Alzheimer's disease $\left(-30 \%,{ }^{16}-18\right.$ to $-51 \%,{ }^{5}-28$ to $-60 \%,{ }^{6}$ Parkinson's disease $(-30$ to $\left.-53 \%,{ }^{5}\right)$ or elderly Down's Syndrome $\left(-44 \%,{ }^{18}\right)$. On average, in the extracerebellar regions the extent of $\mathrm{AChE}$ loss paralleled the degree of ChAT reduction with the reduction in $\mathrm{AChE}$ being slightly less than the ChAT deficit. This observation is similar to the neurochemical findings in necropsy brain studies of other neurogenerative disorders having brain cholinergic loss, namely Alzheimer's disease ${ }^{5}{ }^{6}$ and Parkinson's disease with dementia ${ }^{5}$ in which the cerebral cortical AChE activity (vs. ChAT) is less extensively reduced. Most likely, this is explained by the localisation of $\mathrm{AChE}$ to both cholinergic and noncholinergic neurons. ${ }^{37}$

Recent biochemical data suggest that the AChE reduction in both Alzheimer's disease and Parkinson's disease cerebral cortex is primarily due to a loss of one particular molecular form (intermediate 10S) of the enzyme which may be localised especially to cholinergic neurons. ${ }^{538}$ In this regard it will be of special interest to determine in future studies whether a selective loss of this predominantly membrane bound ${ }^{38}$ form of AChE also occurs in OPCA brain.

In conclusion, the present investigation provides additional neufpchemical evidence suggestive of a marked cortical cholinergic denervation in patients from one well established hereditary OPCA family. OPCA can now be considered to be one of many neurodegenerative disorders, including Alzheimer's disease, ${ }^{14-61617}$ Parkinson's disease with dementia $^{5}$ and elderly Down's Syndrome ${ }^{18}$ having a brain cholinergic reduction. Neurobehaviouralneuropathological-biochemical correlations in prospective studies of affected patients from this OPCA pedigree will be required to assess the clinical significance of our findings. We further suggest that the results of such investigations of non-Alzheimer's disease neurodegenerative disorders having cortical cholinergic deficiency may actually prove more fruitful in providing a greater understanding of the pathophysiological role of the cholinergic system in dementia than studies devoted solely to Alzheimer's disease.

The authors gratefully acknowledge the financial assistance of the Medical Research Council of Canada (grant \# MA-9970) and the National Ataxia Founda- tion (USA). SK is a Career Scientist of the Ontario Ministry of Health.

Presented in part at the Second IBRO World Congress of Neuroscience, Budapest, 16-21 August, 1987.

\section{References}

1 Davies $P$. Neurotransmitter-related enzymes in senile dementia of the Alzheimer type. Brain Res 1979;171:319-27.

2 Perry EK, Perry RH. The Cholinergic System in Alzheimer's disease. In: Roberts PJ, ed. Biochemistry of Dementia, Chichester: John Wiley \& Sons Ltd., 1980;135-83.

3 Rossor MN, Garrett NJ, Johnson AL, Mountjoy CQ, Roth M, Iversen LL. A post-mortem study of the cholinergic and GABA systems in senile dementia. Brain 1982;105:313-30.

4 Pope A, Hess HH, Lewin E. Studies on the microchemical pathology of human cerebral cortex. In: Cohen MM, Snider RS, eds. Morphological and Biochemical Correlates of Neural Activity. New York: Harper Row Publishers, 1964;98-111.

5 Perry EK, Curtis M, Dick DJ, et al. Cholinergic correlates of cognitive impairment in Parkinson's disease: comparisons with Alzheimer's disease. J Neurol Neurosurg Psychiatry 1985;48:413-21.

6 DeKosky ST, Scheff SW, Markesbery WR. Laminar organization of cholinergic circuits in human frontal cortex in Alzheimer's disease and aging. Neurology 1985;35:1425-31.

7 Sims NR, Bowen DM, Davison AN. [14C]Acetylcholine synthesis and [14C] carbon dioxide production from [U-14C]glucose by tissue prisms from human neocortex. Biochem J 1981;196:867-76.

8 Perry EK. The cholinergic hypothesis-ten years on. Br Med Bull 1986;42:63-9.

9 Bartus RT, Dean RC, Beer B, Lippa AS. Cholinergic hypothesis of geriatric memory dysfunction. Science 1982;217:408-17.

10 Kish SJ, Currier RD, Schut L, Perry TL, Morito CL. Brain cholineacetyl-transferase reduction in dominantly inherited olivopontocerebellar atrophy. Ann Neurol 1987;22:272-5.

11 Konigsmark BW, Weiner LP. The olivopontocerebellar atrophies: a review. Medicine 1970;49:227-41.

12 Schut JW. Hereditary ataxia: Clinical study through six generations. Arch Neurol Psychiatry 1950;168:75-95.

13 Wainer BH, Levey AI, Mufson EJ, Mesulam MM. Cholinergic systems in mammalian brain identified with antibodies against choline acetyl-transferase. Neurochem Int 1984;6:163-82.

14 Fibiger HC. The organization and some projections of cholinergic neurons of the mammalian forebrain. Brain Res Rev 1982;4:327-88.

15 Struble RG, Lehmann J, Mitchell SJ, et al. Basal forebrain neurons provide major cholinergic innervation of primate neocortex. Neurosci Lett 1986;66:215-20.

16 Meier-Ruge W, Iwangoff P, Reichlmeier K. Neurochemical enzyme changes in Alzheimer's and Pick's disease. Arch Gerontol Geriatr 1984;3:161-5.

17 McGeer EG, McGeer PL, Kamo H, Tago H, Harrop R. Cortical metaboltsm, acetylcholinesterase staining and pathótogical changes in Alzheimers disease. Can J Neurol Sci 1986;13: 511-6.

18 Perry EK, Perry RH, Smith CJ. Cholinergic receptors in cognitive disorders. Can J Neurol Sci 1986;13:521-7.

19 Ruberg M, Rieger F, Villageois A, Bonnet AM, Agid Y. Acetylcholinesterase and butyrylcholinesterase in frontal cortex and cerebrospinal fluid of demented and non-demented patients with Parkinson's Disease. Brain Res 1986;362:83-91.

20 Greenfield S. Acetylcholinesterase may have novel functions in the brain. Trends in Neurosci 1984;October:364-8.

21 Gray RC, Oliver CP. Marie's hereditary cerebellar ataxia 
(olivoponto-cerebellar atrophy). Minn Med 1941;24:327-35.

22 Haines JL, Schut LJ, Weitkamp LR, Thayer M, Anderson VE. Spinocerebellar ataxia in a large kindred: age at onset, reproduction, and genetic linkage studies. Neurology 1984;34:1542-8.

23 Rich SS, Wilkie PJ, Schut L, Vance G, Orr HT. Spinocerebellar ataxia: Localization of an autosomal dominant locus between two markers on human chromosome 6. Am J Human Genet 1987;41:524-31.

24 Currier R. A classification of ataxia. Ital J Neurol Sci 1984;4: 55-64.

25 Ellman GL, Courtney KD, Andres V, Featherstone RM. A new and rapid colorimetric determination of acetylcholinesterase activity. Biochem Pharmacol 1961;7:88-95.

26 Bonham JR, Gowenlock AH, Timothy JAD. Acetylcholinesterase and butyrylcholinesterase measurement in the pre-natal detection of neural tube defects and other fetal malformations. Clin Chim Acta 1981;115:163-70.

27 Fonnum F. A rapid radiochemical method for the determination of choline acetyltansferase. $J$ Neurochem 1975;24:407-9.

28 Atack JR, Perry EK, Bonham JR, Candy JM, Perry RH. Molecular forms of acetylcholinesterase and butyrylcholinesterase in the aged human central nervous system. J Neurochem 1986;47:263-77.

29 Kan KSK, Chao LP, Forno LS. Immunohistochemical localisation of choline acetyltransferase in the human cerebellum. Brain Res 1980;193:165-71.

30 Kanazawa I. Neurotransmitters and the cerebellum. In: Sobue I, ed. $T R H \&$ Spinocerebellar Degeneration, Amsterdam: Elsevier Science Publishers BV, 1986:105-14.

31 Kasa P, Silver A. The correlation between choline acetyl- transferase and acetylcholinesterase activity in different areas of the cerebellum of rat and guinea pig. $J$ Neurochem 1969;16:389-96.

32 Koeppen AH, Barron KD. The neuropathology of olivopontocerebellar atrophy. In: Duvoisin RC, Plaitakis A, eds. The Olivopontocerebellar Atrophies. New York: Raven Press, 1984:13-38.

33 Landis DMD, Rosenberg RN, Landis SC, Schut L, Nyhan WL Olivopontocerebellar degeneration, clinical and ultrastructural abnormalities. Arch Neurol 1974;31:295-307.

34 Kanazawa I, Kwak S, Sasaki H, et al. Studies on neurotransmitter markers and neuronal cell density in the cerebellar system in olivopontocerebellar atrophy and cortical cerebellar atrophy. J Neurol Sci 1985;71:193-208.

35 Tagliavini F, Pilleri G. Neuronal loss in the basal nucleus of Meynert in a patient with olivopontocerebellar atrophy. Acta Neuropathol 1985;66:127-33.

36 Mesulam MM, Mufson EJ, Levey AI, Wainer BH. Cholinergic innervation of cortex by the basal forebrain: cytochemistry and cortical connections of the septal area, diagonal band nuclei, nucleus basalis (substantia innominata), and hypothalamus in the rhesus monkey. J Comp Neurol 1983;214: 170-97.

37 Eckenstein J, Sofroniew MV. Identification of central cholinergic neurons containing both choline acetyltransferase and acetylcholinesterase and of central neurons containing only acetylcholinesterase. $J$ Neurosci 1983;3:2286-91.

38 Atack JR, Perry EK, Bonham JR, et al. Molecular forms of acetylcholinesterase in senile dementia of Alzheimer Type: Selective loss of the intermediate (10S) Form. Neurosci Lett 1983;40:199-204. 